\title{
Cardiac Resynchronisation Therapy: The Role of Echocardiography in Patient Selection and Follow-up
}

\section{Author: Shirley Middlemost}

Private Cardiologist, MBBCH, FCP(SA), PHD(WITS), FACC

Address for correspondence:

Dr S Middlemost

Vergelegen Mediclinic

Main Road

Somerset West

$7 \mid 30$

Email:

shirley@helderbergheart.co.za

ABSTRACT Resynchronisation of segmental left ventricular mechanics as well as re-coordination of both atrioventricular and interventricular mechanics are potential mechanisms for the clinical improvement observed in patients undergoing cardiac resynchronisation therapy (CRT) for heart failure. Resynchronisation therapy is approved in patients with refractory heart failure symptoms despite optimal medical therapy. The presence of a prolonged QRS duration has been the main criterion used to identify ventricular dyssynchrony. However, based on the current selection criteria, about $20 \%$ to $30 \%$ of patients do not improve after biventricular pacing. Using echocardiography, dyssynchrony may be absent in some heart failure patients with a wide QRS, or present in those with a normal QRS interval.

Echocardiography, and especially the more sophisticated techniques based on Tissue Doppler Imaging, may improve patient selection by identifyinginter-andintra-ventricular dyssynchrony.Echocardiography can also be used to optimise lead placement. Following pacemaker implantation, various echocardiographic techniques can be used to ensure optimal cardiac resynchronisation and to monitor improvements in left ventricular function and hemodynamics. In this review, the different echocardiographic approaches to predict patient response to CRT are discussed. In addition, the use of echocardiography to guide lead positioning and to optimise pacemaker settings following pacemaker implantation is discussed.

Heart failure is the major cause of mortality, morbidity and hospitalisation in patients age $>60$ years ${ }^{(1)}$. Despite major advances in medical therapy, morbidity and mortality remain high. Cardiac resynchronisation therapy (CRT) has been found to provide significant clinical benefit in patients with impaired left ventricular (LV) function, prolonged QRS duration and New York Heart Association (NYHA) functional class III/IV heart failure despite optimal drug therapy ${ }^{(2,3)}$. Studies have shown that CRT improves NYHA functional class, exercise capacity, quality of life and LV systolic function. This is accompanied by a reduction in hospitalisation and a significant improvement in survival, as confirmed in the CARE-HF study ${ }^{(4)}$.
Despite these astonishing results, clinicians have only begun to understand the mechanisms responsible for improvement. Clinicians are struggling to identify those most and least likely to derive benefit from CRT and continue to perfect the technique. Currently, patients who remain significantly symptomatic despite optimal medical therapy are selected mainly on electrocardiogram (ECG) criteria (QRS interval $>120 \mathrm{~ms})^{(5)}$. However, using these selection criteria, about 20\% to $30 \%$ of patients do not respond to CRT. Different factors may influence the ability of CRT to improve clinical outcome, such as patient selection, device programming, lead position and various arrhythmias that result in intermittent CRT delivery.

A major limitation has been the utilisation of the QRS duration as the only marker for dyssynchrony. However, substantial mechanical dyssynchrony, a major predictor of response to CRT, is not necessarily related to electrical dyssynchrony ${ }^{(6,7)}$. QRS width often does not change following CRT and evidence is conflicting as to whether baseline QRS width predicts who will respond to $C R T^{(8)}$. In addition, substantial dyssynchrony may be present in heart failure patients with a norma $\mathrm{QRS}^{(9,10)}$. The presence of LBBB may only identify about half of all patients with mechanical dyssynchrony ${ }^{(8,1)}$.

Mechanical dyssynchrony is complex and should be evaluated at 3 levels. These include:

I. Atrioventricular (AV) dyssynchrony, which results in delayed contraction between the atria and the ventricles. This may produce systolic mitral and tricuspid regurgitation. When the AV delay is excessive, a gap occurs between the end of atrial filling ( $A$ wave) and the beginning of left ventricular contraction. During this delay, the mitral valve closes passively (soft first heart sound), as the left atrial pressure falls below LV pressure during late diastole, so that diastolic mitral regurgitation may occur. The shortened ventricular filling time limits net diastolic stroke volume ${ }^{(12)}$. Atrial systole occurs during early passive filling and this further reduces LV filling ${ }^{(13)}$. When the AV delay is too short, the left atrial systolic peak pressure occurs after the onset of $L V$ systole. As a result, the left atrial contraction is not able to open the mitral valve because LV pressure greatly exceeds the left atrial peak pressure. The mean left atrial pressure is increased, with possible symptoms of pulmonary congestion.

2. Interventricular dyssynchrony, which is associated with an earlier onset of right ventricular (RV) contraction and ejection, during the LV end-diastolic period.The higher RV pressure displaces the septum into the $L V$, resulting in decreased mechanical performance ${ }^{(14)}$. 
3. Intraventricular dyssynchrony, which is associated with a reduction in ventricular performance and global LV ejection fraction ${ }^{(15)}$. There is wasted work with either early or delayed regional myocardial contraction. The latter may even be delayed to early diastole ${ }^{(16)}$. The early contraction occurs when pressure is low and does not lead to ejection. The late contraction occurs at higher stresses and results in paradoxical stretch of any earlier contracting segments. The net result is a decline in systolic performance ${ }^{(17)}$. Delayed early relaxation shortens LV filling time, whilst delayed papillary muscle contraction contributes to mitral regurgitation.

Considering the limitations of conventional ECG, various echocardiographic techniques, some quite novel, have been developed to determine the extent of mechanical dyssynchrony. These include conventional 2-dimensional (2D) and Doppler echocardiography and newerTissue Doppler methods. There is currently no consensus on the definition of echocardiographically measured dyssynchrony.

\section{CONVENTIONAL ECHOCARDIOGRAPHY}

Pitzalis and colleagues utilized standard M-mode echocardiography to measure the extent of septal-to-lateral wall motion delay as derived from the parasternal short-axis view ${ }^{(8)}$. A delay $>130$ ms has been

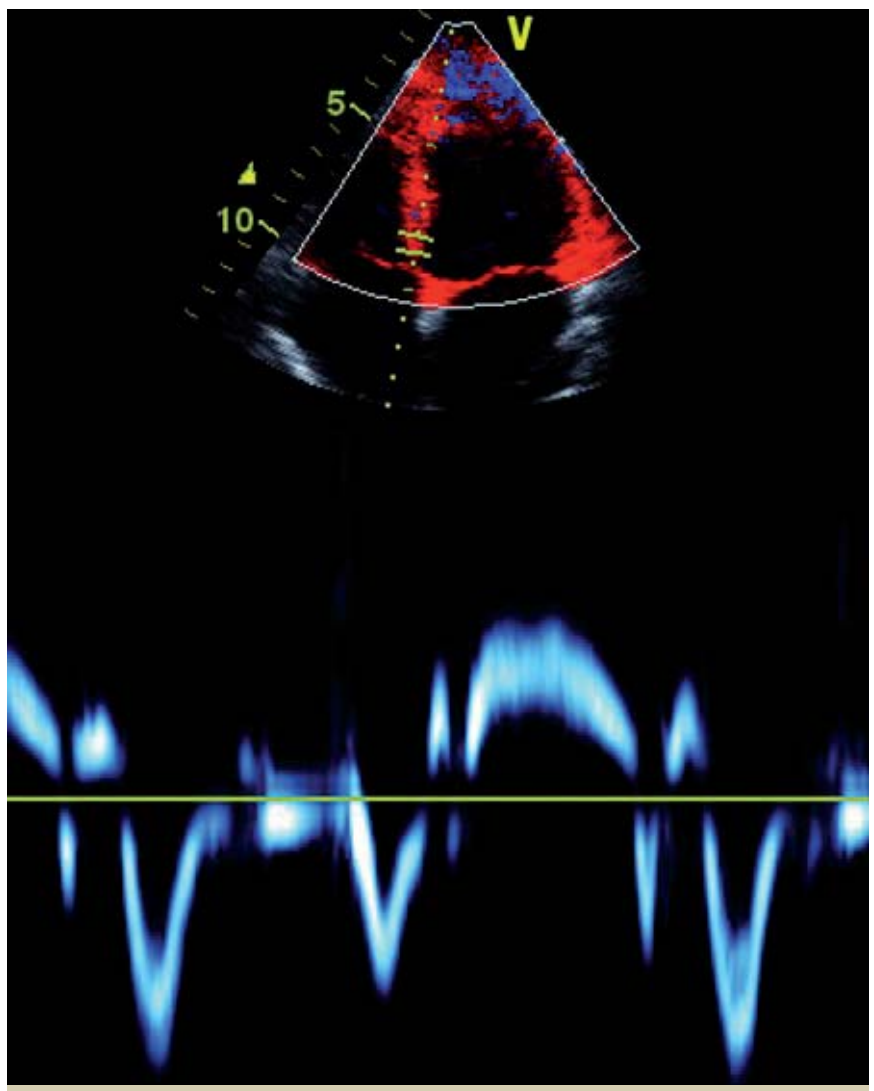

FIGURE 1: Pulse Wave Tissue Velocity Imaging

Pulsed Doppler interrogation measures the instantaneous peak velocities from the myocardium which passes through the sample volume during the cardiac cycle. The sharp narrow positive spike represents myocardial velocity during the isovolumic contraction period. This is followed by a broad dominant positive waveform representing myocardial velocity during the ejection phase. There are two dominant negative waveforms (below the line) which represent myocardial velocity during rapid atrial filling (E-wave) and atrial contraction (A-wave), respectively.

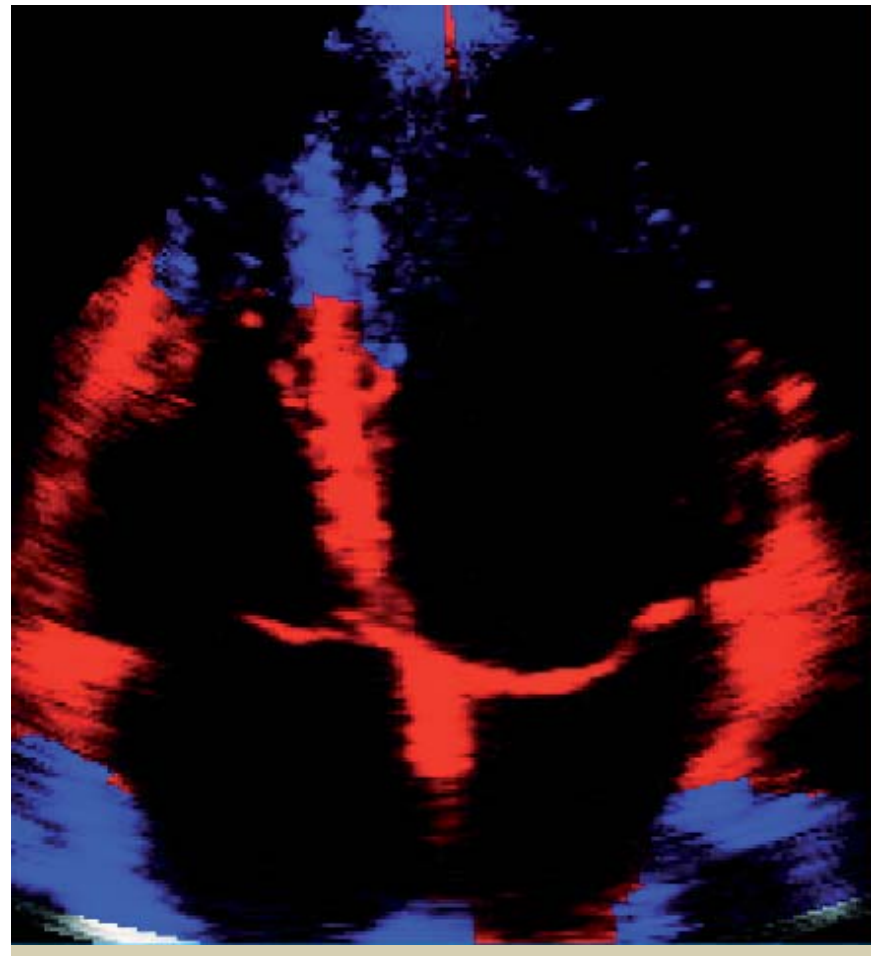

FIGURE 2: Colour Tissue Velocity Imaging

Red colour displays tissue motion towards the probe. Blue colour displays motion away from the probe.

proposed as a marker of intraventricular dyssynchrony and has been shown to be a powerful predictor of reverse remodeling. This index cannot be derived in patients with an akinetic septum or posterior wall and it is not always possible to get perpendicular M-mode sections through the LV.

Interventricular mechanical delay (IVMD) can also be determined using pulsed Doppler. The time difference between the onset of the pulmonary flow and the aortic flow $>40 \mathrm{~ms}$ is considered indicative of interventricular dyssynchrony ${ }^{(18,19)}$. In the CARE-HF study, a prolonged aortic pre-ejection delay (>140ms) was an additional criterion reflecting interventricular delay in those with a QRS interval between $120 \mathrm{~ms}-150 \mathrm{~ms}^{(4)}$.

\section{TISSUE DOPPLER IMAGING (TDI),TISSUE- TRACKING, STRAIN AND STRAIN RATE}

With the limited role of conventional echocardiography in the assessment of cardiac dyssynchrony, several newer echocardiographic applications have emerged with potential application in CRT. These include the following:

\section{Tissue Velocity Imaging}

Tissue Velocity Imaging (TVI) uses myocardial Doppler frequency shifts to quantify regional myocardial function by measuring segmental peak systolic and diastolic tissue velocities. TVI can be displayed as pulsed Doppler (Figure I), colour Doppler (Figure 2) and colour M-mode. The 2D colour TVI image can be converted to TVI waveforms to allow further off-line quantitative analysis (Figure 3). 


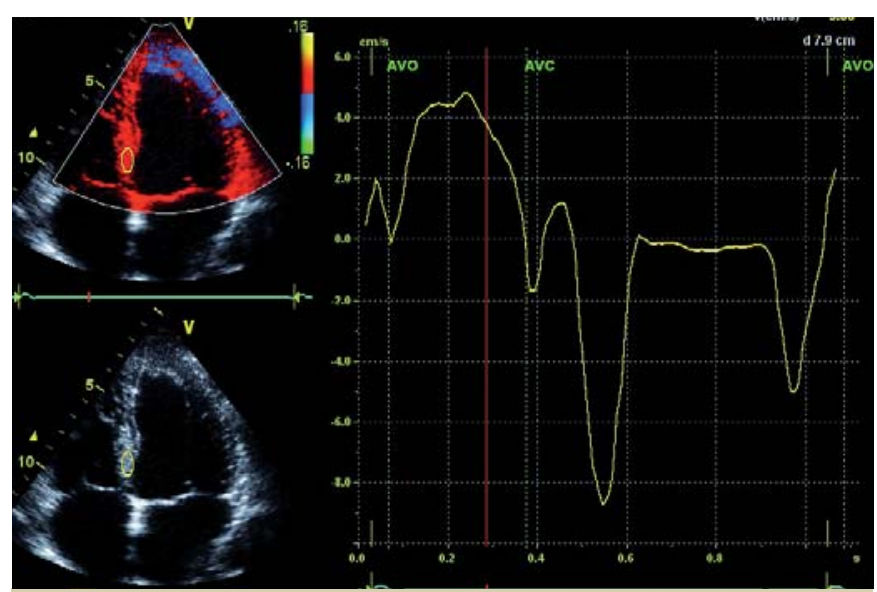

FIGURE 3: The regional velocity sampled from the basal septum in this normal subject is displayed in the diagram on the right. Conventionally, systolic motion of segments towards the apex (and hence the transducer) is ascribed a positive value while shortening of a segment is ascribed a negative value. Aortic valve opening and closure are displayed to enable accurate timing of mechanical events within the cardiac cycle.

Interventricular dyssynchrony has been defined by the difference in time-to-peak myocardial velocities between one RV segment and the mean of $6 \mathrm{LV}$ myocardial segments in the apical 4-chamber, 2-chamber and long-axis views ${ }^{(20)}$.

Intraventricular dyssynchrony is present when there is a delay of $>60 \mathrm{~ms}$ in the time to onset of peak systolic velocities from the QRS complex using a 2-sample model (basal septum and basal lateral wall) ${ }^{(21,22) . ~ I n ~ a ~ m o r e ~ c o m p l e x ~ a n d ~ t i m e-c o n s u m i n g ~ 12-s e g m e n t ~ m o d e l, ~}$ derived from the 6 basal and 6 mid-ventricular segments, ${ }^{(10)} Y u$ and colleagues defined intraventricular dyssynchrony as present when:

a) there was a $>100 \mathrm{~ms}$ difference between the peak systolic velocities of any 2 of 12 LV segments or b) the SD (standard deviation) of all time intervals measuring time to peak systolic velocity in 12 LV segments was $>33 \mathrm{~ms}$.

\section{Tissue Tracking}

Although TVI is used to identify areas of dyssynchrony, it is limited by the fact that it does not allow simultaneous comparison of regional timing in different segments within one cardiac cycle. Tissue Doppler measures the velocity at a single point and cannot discriminate between passive myocardial motion as a result of tethering and active myocardial contraction $^{(23)}$. TVI technology includes tissue tracking and strain rate imaging. Tissue tracking is performed in the apical view and allows the measurement and visualisation of longitudinal motion in each cardiac segment during the different phases of the cardiac cycle.This is done by integrating tissue velocities to yield tissue displacement, which measures the total distance that particular point travels. Tissue Tracking is displayed as a colour band representing motion during systole. Each point in the myocardium is colour coded, with displacement occurring from enddiastole to end-systole. The width of each colour bar is a measure of regional strain over the time period interrogated (Figure 4).

Tissue synchronisation imaging (TSI) is a parametric imaging tool derived from 2-dimensional (2D) TVI images. It automatically calculates and colour-codes the time-to-peak tissue velocity in every portion in the image, with reference to the QRS signal.The TSI algorithm detects positive velocity peaks within a specified time interval. The colour coding ranges from green (earliest), yellow, orange, to red (latest) within this interval. With TSI, the colour represents the amount of tissue motion delay rather than the absolute value of the tissue velocity. The variation in colour provides both a quantitative and qualitative

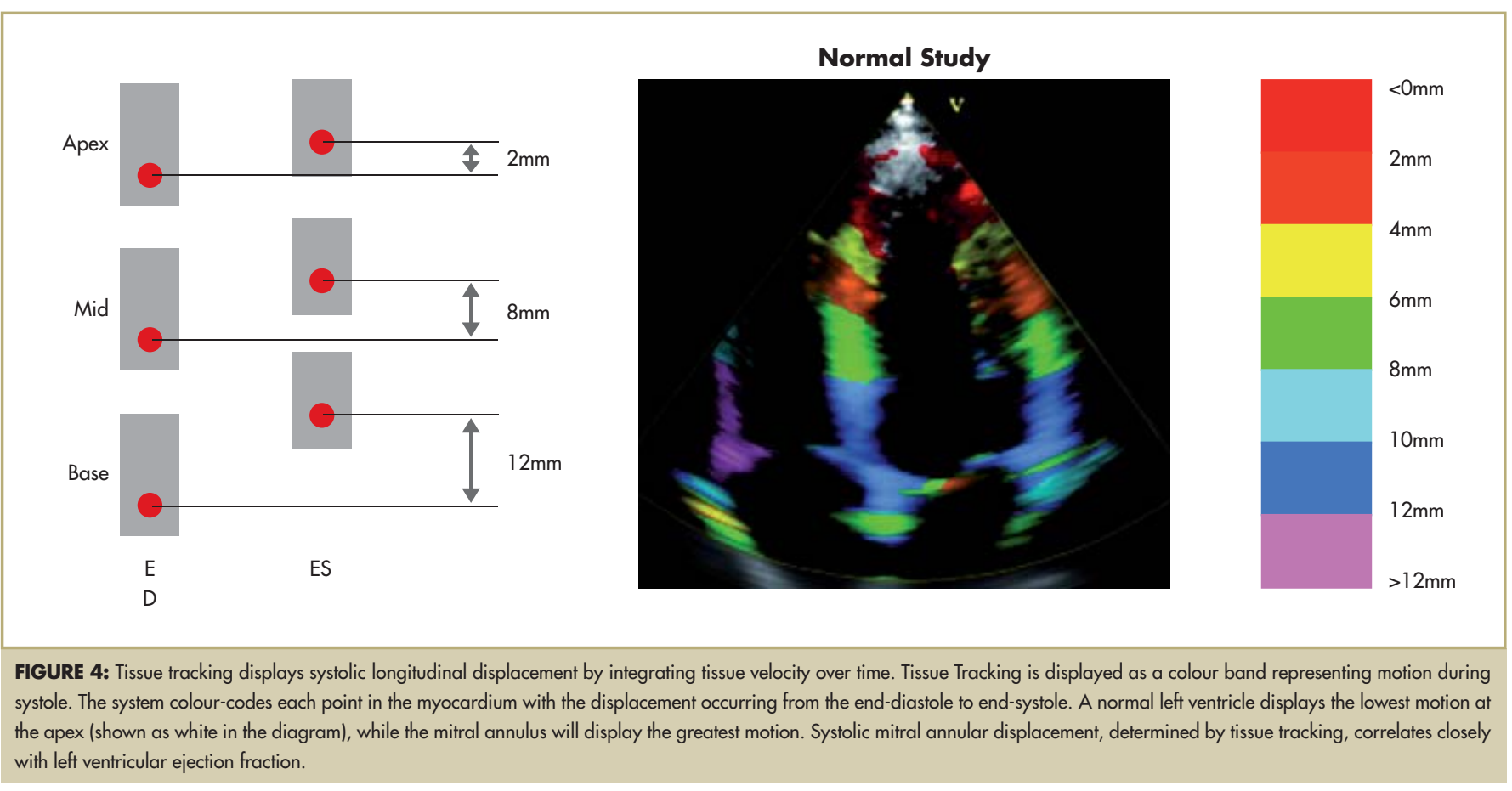




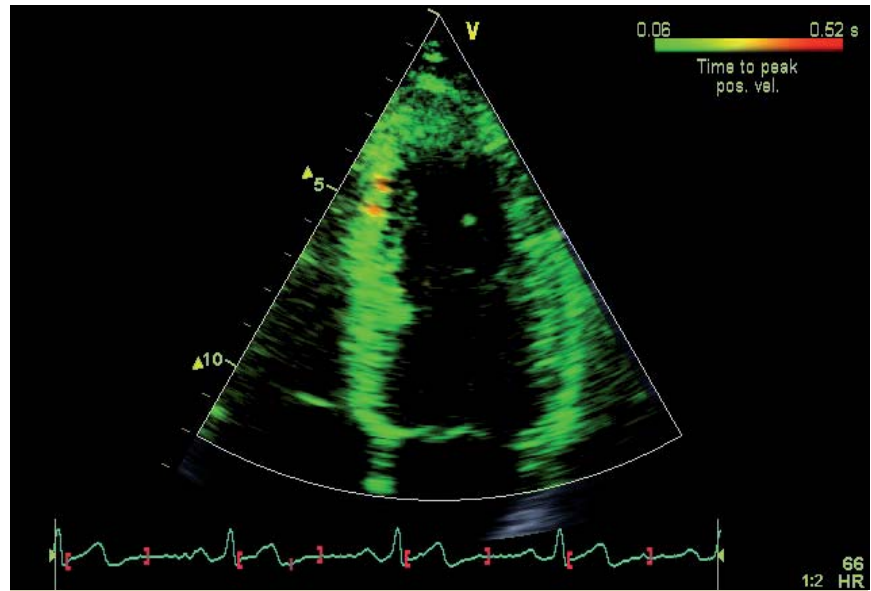

FIGURE 5: Tissue Synchronized Imaging is a parametric imaging tool based on Tissue Velocity Imaging that provides clinicians with additional image enhancement for assessing delayed cardiac motion. The TSI parametric image analyzes the tissue velocity signals within a specified portion of the cardiac cycle. Since these peaks will occur in relation to overall motion, delayed wall motion will produce a delayed peak velocity. The amount of delay within a defined area of the cardiac cycle, indicated by the markers on the ECG, is used to assign or map a colour to that location in the image. The colour represents the amount of tissue motion delay rather than the absolute value of the tissue velocity. The variation in colour provides both a qualitative and quantitative representation of wall motion delay, allowing easy and rapid identification and evaluation of asynchronous wall motion. The figure depicts a normal study with all regions shown in green, reaching peak velocity at the same time early in systole.

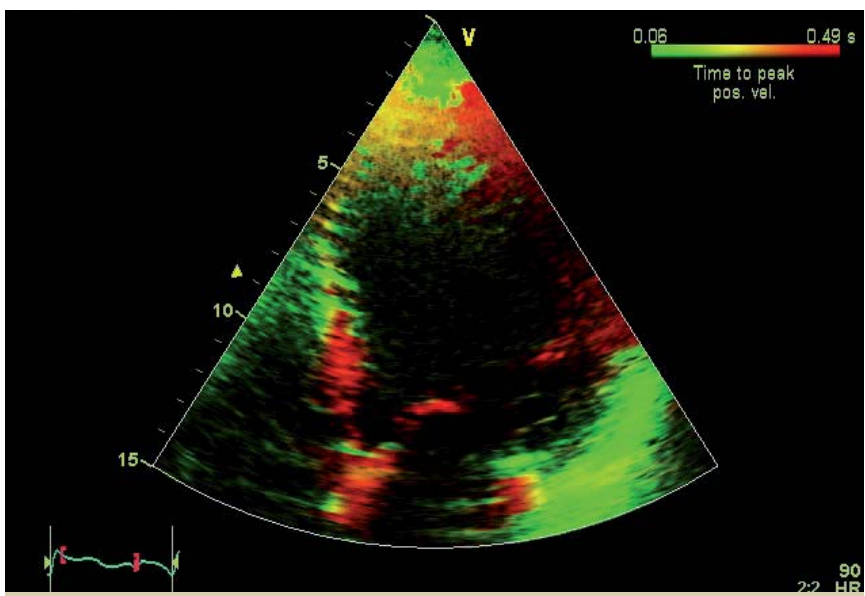

FIGURE 6: Utilizing Tissue Synchronized Imaging, it is easy to appreciate the dyssynchronous regions in the left ventricle. The regions which reach peak velocities at different times have different colours, with the red areas being most delayed.

representation of wall motion delay, allowing immediate identification of regional delay in systole by comparing the colour mapping of orthogonal walls (Figure 5 and 6)(24,25).

Various TSI parameters have been used to predict an improvement in clinical outcome. Murphy, et al. used TSI to identify the area of latest peak systolic velocity before CRT. Pacing at this site resulted in the greatest clinical and hemodynamic improvement ${ }^{(26)}$. Whilst $Y u$, et al, confirmed that the time-to-peak systolic velocity (Ts) measured in the ejection phase in 12 LV segments, derived in the apical view, was the best modality to predict reverse LV remodeling after CRT. When the lateral wall is delayed, the patient has a high likelihood of response to
CRT ${ }^{(27)}$. In the absence of lateral wall delay, patients unlikely to respond to CRT were identified when the systolic synchronicity index, defined as the standard deviation of the peak tissue velocity in $12 \mathrm{LV}$ segments in the ejection phase, was $<34.4 \mathrm{~ms}$.

Although this study did not find that post-systolic shortening (PSS), defined as contraction after aortic valve closure, was useful for the identification of responders, others have shown that, in non-ischemic heart failure, the presence of PSS in more than $20 \%$ of the basal segments on all three apical views was predictive of clinical improvement ${ }^{(28)}$.

\section{Strain and Strain Rate}

The temporal analysis of myocardial motion by velocity imaging allows no definite conclusions about the timing of regional contraction. As already discussed, velocity imaging cannot differentiate passive myocardial motion related to tethering artefacts or cardiac translation from active myocardial contraction. To overcome the limitations of velocity imaging, strain and strain rate imaging have been developed to identify the regional deformation properties of the myocardium. The information is derived from tissue Doppler velocity data and measures the timing and extent of myocardial deformation. Strain measures the change in shape of myocardial segments and strain rate imaging measures how fast the change has occurred (Figures 7, 8). Strain is defined as the change in length normalized to the initial length of the myocardium and is therefore expressed as a percentage. Strain measures the activity of 2 points within the myocardium. Because the length at peak systole is shorter than the original length, strain (and strain rate) has a negative polarity when measured in the longitudinal axis ${ }^{(29)}$. The opposite occurs in the short-axis plane (radial) when the myocardium thickens during systole so that strain and strain rate have a positive polarity.

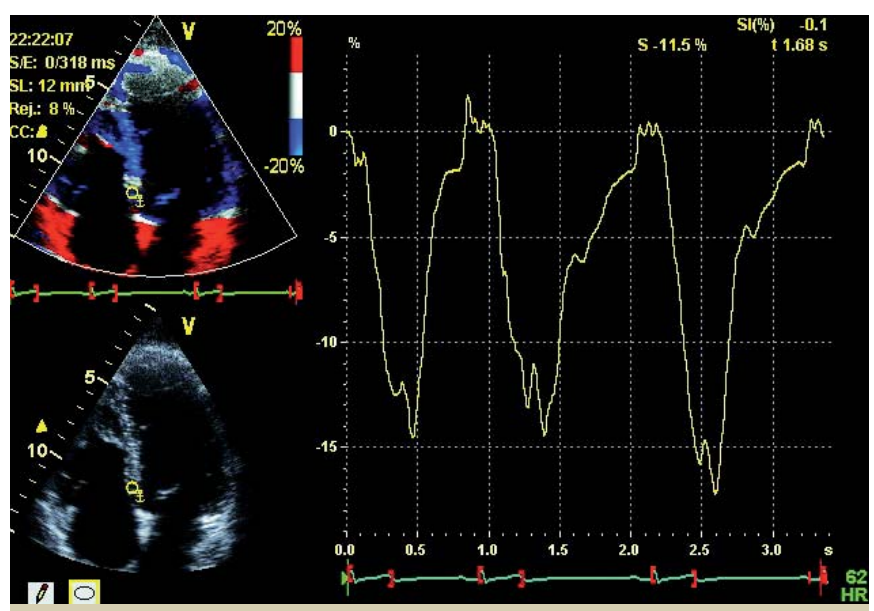

FIGURE 7: Strain Imaging provides regional detection of myocardial contraction. Strain measures the percent of regional deformation of the myocardium (percentage change in length). Systolic motion of the segments towards the apex (towards the transducer) is ascribed a positive value and shortening of the segment a negative strain value. The opposite will apply to regional lengthening. The diagram depicts a normal study with negative strain during systole in the basal septum. There is no lengthening of the myocardial segment during this time interval. 


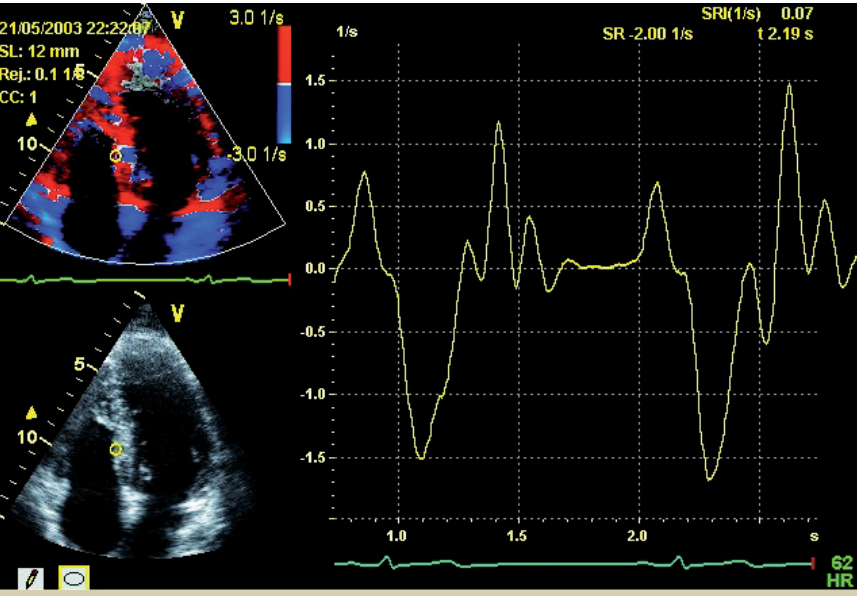

FIGURE 8: Strain rate measures the speed of deformation (change in shape) of the myocardium. As with Strain Imaging, systolic motion of the segments towards the apex (towards the transducer) is ascribed a positive value and shortening of the segment a negative strain rate value. Simultaneous ECG recordings allow correct timing of events. This normal study depicts a negative systolic Strain Rate in the basal septum.

Strain and strain rate imaging measure myocardial deformation in systole and are able to identify reliably the presence of delayed contractions and thus the presence of a recruitable contractile reserve that can be utilized by CRT, by transferring it from the pre or postejection period to the systolic ejection phase. Ischemic myocardial segments can also display PSS. These delayed segments would not be expected to respond to CRT.

\section{REAL-TIME THREE-DIMENSIONAL}

\section{ECHOCARDIOGRAPHY}

This novel imaging modality allows functional assessment of all 16 individual segments of the LV. A cast of the LV is obtained and regional volume curves are obtained. A dyssynchrony index is obtained by calculating the time taken to each minimal volume for each segment as a percentage of the cardiac cycle. This is expressed as a percentage of the duration of the cardiac cycle to allow comparison between patients with different heart rates. Using this technique, it has been shown that mechanical dyssynchrony is increasingly prevalent with worsening LV function, independently of QRS duration ${ }^{(9)}$.

\section{ECHOCARDIOGRAPHY AND LV LEAD PLACEMENT}

One of the challenges for the echocardiographer is to identify the optimal LV segment for placement of the LV lead. Pacing in the "wrong" area may account for a failed response in appropriately selected patients. Moreover, incorrect lead placement may be detrimental and lead to further dyssynchrony ${ }^{(30)}$.
Using tissue Doppler strain imaging, a recent study has confirmed that pacing at the site of maximal mechanical delay is associated with reverse remodeling, (26) a powerful predictor of a favourable clinical outcome(28,31). Echocardiography could be helpful at the time of implantation to optimise lead position. Ongoing clinical trials seek to address this issue.

\section{POST-IMPLANTATION USE OF}

\section{ECHOCARDIOGRAPHY FOR FOLLOW-UP AND OPTIMISATION}

The three primary aspects of programming or optimisation of resynchronisation therapy that have become salient to management have been the optimisation of atrio-ventricular (AV) delay, mechanical synchrony and interventricular timing. Although much attention has focused on the optimisation of the AV delay, it has become evident that optimisation of mechanical synchrony may be the most important parameter to target as an endpoint to ensure successful therapy.

\section{Optimising Atrio-Ventricular Delay}

Although AV-delay optimisation can clearly impact hemodynamics in patients undergoing $C R T,{ }^{(32,33)}$ it has less influence on LV function than the optimal pacing site ${ }^{(34)}$. An optimal AV delay is that which allows completion of the end-diastolic filling flow prior to ventricular contraction, thereby providing the longest diastolic filling time. The AV interval can be optimised using 2 methods:

I. calculating the AV-delay interval from pulsed Doppler measurement of the timing of mitral valve closure at a short and long AV-delay setting (mitral inflow method) ${ }^{(35)}$

2. determining the AV-delay that produces the greatest increase in the aortic velocity time integral (VTI) using continuous wave Doppler. A recent study has shown that the aortic VTI method may be superior to the mitral inflow method in patients with a QRS interval $>150 \mathrm{~ms}$. ${ }^{(36)}$

Optimisation of the Ventricular-to-Ventricular (VV)-Interval In patients with heart failure and significant conduction delay undergoing CRT, simultaneous stimulation of the LV and right ventricle (RV) may not be appropriate. Therefore, altering LV-RV stimulation by varying durations may further optimise LV resynchronisation. The benefit of sequential versus simultaneous biventricular pacing (BVP) has been evaluated in a small study of 21 patients ${ }^{(37)}$. This study used TDI with tissue tracking pre-operatively to identify regions displaying delayed longitudinal contraction. Post-implantation, it was confirmed that 
individually tailored sequential rather than simultaneous BVP resulted in an additional increase in diastolic filling time, with a simultaneous significant increase in LV ejection fraction from $30+5 \%$ to $34+6 \%$. It has been recommended that the $\mathrm{V}$-interval be optimised before the $\mathrm{AV}$ interval, to prevent inappropriate AV-delay programming, which might reduce $L V$ filling ${ }^{(33)}$.

\section{Assessment of Myocardial Remodeling}

Myocardial remodeling is a complex process and not well understood. Positive remodeling is defined as a $>15 \%$ reduction in $\mathrm{LV}$ end-systolic volume $^{(28,31)}$. This has been observed in about $54 \%-64 \%$ of patients at 3 months follow-up ${ }^{(38,39)}$. Echocardiography has also demonstrated a reduction in the severity of mitral regurgitation in CRT responders. This is related, in part, to resynchronisation of papillary muscle contraction that restores mitral subvalvular size and function towards normal and improved atrioventricular coupling ${ }^{(3)}$. The reduction in MR can be seen almost immediately after optimal CRT.

\section{EVALUATING CRT “NON-RESPONDERS”}

If CRT has not achieved its anticipated response, the following questions should be addressed:

I. Has the physiology of CRT been optimised for this patient? Has diastolic timing been optimally adjusted? Has systolic resynchronisation been fully enhanced? All of these issues can be addressed using echocardiography.

2. Is CRT being consistently delivered? Are the ventricles being consistently paced at all physiologic heart rates? Is the LV stimulus consistently suprathreshold? Is there consistent atrial and ventricular sensing? Answering these questions requires an understanding of how to use diagnostics from the CRT device.

\section{FUTURE DIRECTIONS}

Some potential issues that may be addressed include:

I. The role of echocardiography in the catheterisation laboratory to guide LV lead placement. It has been shown that, in patients with LBBB, RV apical pacing alters the activation wavefront of the LV compared with intrinsic rhythm ${ }^{(40)}$. Perhaps echocardiography can be used in the catheterisation laboratory to ensure that RV pacing has not altered the LV segment with dyssynchrony. Also, the separate effects of RV, LV and BVP can be assessed.
2. The role of regional myocardial infarction on long-term clinical outcomes and whether echocardiography can address these issues.

3. The role of CRT in patients with heart failure and an $E F<35 \%$ but with a QRS duration $<120 \mathrm{~ms}$ or a right bundle branch block pattern on ECG.

4. The role of CRT in those with dyssynchrony but with less severe symptoms of heart failure (NYHA functional class II) or with less severe LV dysfunction (EF 35\% - 40\%).

\section{CONCLUSIONS}

Current guidelines recommend CRT in patients with reduced LV ejection fraction, a prolonged QRS interval and severe heart failure symptoms despite optimal medical therapy. Based on these selection criteria, about one third of patients fail to respond. Echocardiography can be used to improve patient selection and should be included as part of the workup to identify potential candidates for CRT. In addition, echocardiography has an important role in the identification of the optimal site for LV lead placement and in the optimisation of CRT programming with regard to the $\mathrm{AV}$ - and $\mathrm{V}$ - intervals. Echocardiography also allows assessment of resynchronisation and follow-up of patients after CRT.

\section{ACKNOWLEDGEMENTS}

All the graphics in this paper have been supplied with the kind permission of GE Healthcare. Personal thanks are extended to the product manager of MEDHOLD GEMS, Mr Dave Evans, for all his professional assistance. 


\section{REFERENCES:}

I. Cleland JGF. The heart failure epidemic: Exactly how big is the problem? Eur Heart J 2001;22:623-626

2. Cazeau S, Leclercq C, Lavergne T, et al. Investigators. FtMCSiCS: Effects of multisite biventricular pacing in patients with heart failure and intra-ventricular conduction delay N Engl J Med 2001;344;873-880

3. Abraham W, Fisher WG, Smith AL, et al. For the MIRACLE Study Group: Cardiac resynchronisation in heart failure. N Engl J Med 2002;346: I 845- | 853)

4. Cleland JGF, Daubert JC, Erdmann E, et al. CARE-HF. N Engl J Med 2005;352:15391548

5. ACC/AHA Guidelines Update for Management of Chronic Heart Failure in the Adult. J Am Coll Cardiol 2005;46: | | |6- | | 43

6. Leclercq C, Faris O,Tunin R, et al. Systolic improvement and mechanical resynchronisation does not require electrical synchrony in the dilated failing heart with left bundle branch block. Circulation 2002; 106: 1760-1763

7. Kass DA. Predicting cardiac resynchronisation therapy response by QRS duration: the long and the short of it. J Am Coll Cardiol 2003;42:2125-2127

8. Pitzalis MV, lacoviello M, Romito R, et al. Cardiac resynchronisation therapy tailored by echocardiographic evaluation of ventricular synchrony. J Am Coll Cardiol 2002;40:16151622

9. Kapetanankis S, Kearney MT, Siva A, et al. Real-time three dimensional echocardiography: A novel technique to quantify global left ventricular mechanical dyssynchrony. Circulation 2005; 1 12:992-1000

10. Yu CM, Lin H, Zhang Q, et al. High prevalence of left ventricular systolic and diastolic asynchrony in patients with congestive heart failure and normal QRS duration. Heart 2003:89:54-60

I I. Yu CM, Yang H, Lau CP, et al. Regional left ventricular mechanical asynchrony in patients with heart disease and normal QRS duration: implication for biventricular pacing therapy. Pacing Clin Electrophysiol 2003;26(pt I):562-570

12. Brecker SJ, Xiao HB, Sparrow J, Gibson DG. Effects of dual-chamber pacing with short atrioventricular delay in dilated cardiomyopathy. Lancet 1992;340:21 17-2124

13. Kass DA. Ventricular dyssynchrony and mechanisms of resynchronisation therapy. Eur Heart | 2002;4:D23-30

14. Grines CL, Bashore TM, Boudoulas H, et al. Functional abnormalities in isolated left bundle branch block: the effect of interventricular asynchrony. Circulation 1989;79:84553

15. Prinzen FW, Augustijn $\mathrm{CH}$, Arts $\mathrm{T}$, et al. Redistribution of myocardial fiber strain and blood flow by asynchronous activation. Am J Physiol 1990;259:H300-308

16. Nelson GS, Curry CW,Wyman BT, et al. Predictors of systolic augmentation from left ventricular preexcitation in patients with dilated cardiomyopathy and intraventricular conduction delay. Circulation 2000; 10 1:2703-2709

17. Park RC, Little WC, O'Rourke RA. Effect of alteration of left ventricular activation sequence on the left ventricular end-systolic pressure-volume relation in closed-chest dogs. Circ Res 1985:57:706-717

18. Porciani MC, Puglisi A, Colella A, et a. Echocardiographic evaluation of the effect of biventricular pacing: the InSync Italian Registry. Eur Heart J Suppl 2000;2(suppl J):J2330

19. Rouleau F, Merheb M, Geffroy S, et al. Echocardiographic assessment of the interventricular delay of activation and correlation to the QRS width in dilated cardiomyopathy. Pacing Clin Electrophysiol 200 1;24:1500-1506

20. Turner MS, Bleasdale RA, Vinereanu D, et al. Electrical and mechanical components of dyssynchrony in heart failure patients with normal QRS duration and left bundle-branch block: impact of left and biventricular pacing. Circulation 2004; 109:2544-2549

21. Bax J, Molhoek SG, Van Erven L, et al. Usefulness of myocardial tissue Doppler echocardiography to evaluate left ventricular dyssynchrony before and after biventricular pacing in patients with idiopathic dilated cardiomyopathy. Am J Cardiol 2003;91:94-97

22. Bax JJ, Marwick TH, Molhoek SG, et al. Left ventricular dyssynchrony predicts benefit of cardiac resynchronisation therapy in patients with end-stage heart failure before pacemaker implantation. Am I Cardiol 2003:92:1238-1240

23. Abraham TP, Nishimura RA, Holmes DR Jr, Belohlavek M, Seward JB. Strain rate imaging for assessment of regional myocardial dysfunction results from a clinical model of septa ablation. Circulation 2002; 105: | 403-1406

24. Gorcsan ] III, Kanzaki H, Bazaz R, Dohi K, Schwatzman D. Usefulness of echocardiographic tissue resynchronisation imaging to predict acute response to cardiac resynchronisation therapy. Am J Cardiol 2004;93:1 |78-1 | 8 |
25. Kanzaki H, Jacques D, Sade LE, et al. Regional correlation of color-coded tissue Doppler to quantify improvements in mechanical left ventricular synchrony after biventricular pacing therapy. Am J Cardiol 2003;92:752-755

26. Murphy RT, Sigurdsson G, Mulamalla S, et al.Tissue synchronisation imaging and optimal left ventricular pacing site in cardiac resynchronisation therapy. Am J Cardiol 2006;97:1615-1621

27. Yu CM, Zhang Q, Fung JW, et al. A novel tool to assess systolic asynchrony and identify responders of cardiac resynchronisation therapy by tissue synchronisation imaging. I Am Coll Cardiol 2005;45:677-684

28. Sogaard P, Egeblad H, Kim WY, et al. Tissue Doppler imaging predicts improved systolic performance and reversed left ventricular remodeling during long-term cardiac resynchronisation therapy. J Am Coll Cardiol 2002;40:723-730

29. Abraham TP, Nishimura RA. Myocardial strain: can we finally measure contractility? J Am Coll Cardiol 2001;37:731-734

30. Butter C, Auricchio A, Stellbrink C, et al. Should stimulation site be tailored in the individual heart failure patient? Am J Cardiol 2000;86(suppl): I 44K-I5I K

3I. St. John Suton MG, Plappert T, Abraham WT, et al. Multicenter InSync Randomised Clinical Evaluation (MIRACLE) Study Group. Effect of cardiac resynchronisation therapy on left ventricular size and function in chronic heart failure. Circulation 2003; 107:19851990

32. Auricchio A, Stellbrink C, Block M, et al. Effect of pacing chamber and atrioventricular delay on acute systolic function of paced patients with congestive heart failure. Circulation 1999:99:2993-300।

33. Auricchio A, Ding J, Spinelli JC, et al. Cardiac resynchronisation therapy restores optima atrioventricular mechanical timing in patients with ventricular conduction delay. J Am Coll Cardiol 2002:39:1 163-1169

34. Kass DA, Chen E, Curry C, et al. Improvement in left ventricular mechanics from acute DDD pacing in patients with dilated cardiomyopathy and ventricular conduction delay. Circulation 1999:99:1567-1573.

35. Ritter P, Dib JC, Lelievre T, et al. Quick determination of the optimal AV delay at rest in patients paced in DDD mode for complete AV block (abstr). Eur J CPE 1994:AI63

36. Kerlan JE,Sawhney NS,WaggonerAD, et al. Prospective comparison of echocardiographic atrioventricular delay optimisation methods for cardiac resynchronisation therapy Heart Rhythm 2006;3: I 48-I54

37. Sogaard P,Egeblad H, Pederson A, et al. Sequential versus simultaneous resynchronisation for severe heart failure. Circulation 2002;106:2078-84

38. Yu CM, Fung JWH, Zhang $\mathrm{Q}$, et al. Tissue Doppler imaging is superior to strain rate imaging and postsystolic shortening on the prediction of reverse remodeling in both ischemic and nonischemic heart failure after cardiac resynchronisation therapy Circulation 2004; I 10:66-73

39. Stellbrink C, Breithardt OA, Franke A, et al. Impact of cardiac resynchronisation therapy using hemodynamically optimised pacing on left ventricular remodeling in patients with congestive heart failure and ventricular conduction disturbances. J Am Coll Cardio 2001:38:1957-1965

40. Auricchio A, Fantoni C, Regoli F, et al. Characterisation of left ventricular activation in patients with heart failure and left bundle-branch block. Circulation 2004; 109:1 I331139 